\title{
INFLATIONARY DILATONIC de SITTER UNIVERSE FROM SUPER YANG - MILLS THEORY PERTURBED BY SCALARS AND SPINORS
}

\author{
Iver Brevik ${ }^{1}$ \\ Department of Energy and Process Engineering \\ Norwegian University of Science and Technology \\ N-7491 Trondheim, Norway \\ John Quiroga ${ }^{2}$ \\ Department of Physics \\ Universidad Tecnológica de Pereira \\ Colombia \\ and \\ Lab. for Fundamental Study, \\ Tomsk State Pedagogical University, \\ Tomsk 634041, Russia
}

August 2003

\begin{abstract}
In this paper a quantum $\mathcal{N}=4$ super Yang-Mills theory perturbed by dilaton-coupled scalars and spinor fields, is considered. The induced effective action for such a theory is calculated on a dilaton-gravitational background using the conformal anomaly found via the AdS/CFT correspondence. Considering such an effective action (using the large $\mathrm{N}$ method) as a quantum correction to the classical gravity action with cosmological constant, we study the effect from the dilaton on the scale factor (this corresponds to an inflationary universe without dilaton ). It is shown that, depending on the initial conditions for the dilaton, the dilaton may slow down, or accelerate, the inflation process. At late times, the dilaton is decaying exponentially. Different possible cases corresponding to a dilatonic dS Universe are analyzed with respect to the equations of motion.
\end{abstract}

\footnotetext{
${ }^{1}$ E-mail: iver.h.brevik@mtf.ntnu.no

${ }^{2}$ E-mail: jquiroga@tspu.edu.ru
} 
Recent evidence has shown that the present Universe is subject to accelerated expansion and may thus be entering an inflationary phase now. In view of this, taking into account that the inflationary Universe is currently considered to be a realistic model for the evolution of the very Universe (for a general review, see [1]), one may think about reconsidering quantum cosmology and constructing a new (or modified) version of the theory of the very early Universe.

In the present work, we consider one theory of this kind which has become very popular recently in connection with the AdS/CFT correspondence, namely quantum cosmology as following from $\mathcal{N}=4$ quantum super YM theory. Using conformal anomaly on a dilaton-gravitational background the anomaly induced effective action was constructed in [3], and the consequences it may lead to in the early Universe were discussed in the same reference. The present paper presents the generalization of such a model for quantum cosmology [3] where $\mathcal{N}=4$ SYM is perturbated by quantum scalar and spinor fields interacting with the dilaton. Taking into account that on a purely gravitational background such an effective action leads to the possibility of inflation, we will show that the role of the dilaton is to accelerate, or to slow down, the inflationary expansion, depending on which choice is made for the initial conditions of the dilaton.

Let us start from the Lagrangian of local superconformally invariant $\mathcal{N}=$ 4 super YM theory in the background of $\mathcal{N}=4$ conformal supergravity. The corresponding vector multiplet is $\left(A_{\mu}, \psi_{i}, X_{i j}\right)$. Supposing that super YM theory interacts with conformal supergravity in a $\mathrm{SU}(1,1)$ covariant way and keeping only kinetic terms, we get:

$$
\begin{aligned}
L_{S Y M}= & -\frac{1}{4}\left(e^{-\phi} F_{\mu \nu} F^{\mu \nu}+\tilde{C} F^{\mu \nu} F^{*}{ }_{\mu \nu}\right)- \\
& -\frac{1}{2} \bar{\psi}^{i} \gamma^{\mu} D_{\mu} \psi^{i}-\frac{1}{4} X_{i j}\left(-D^{2}+\frac{1}{6} R\right) X^{i j}+\ldots
\end{aligned}
$$

We note that the scalar $\phi$ from the conformal supergravity multiplet is written as $\tilde{C}+i e^{-\phi}$. We also note that the first term in (11) describes the dilaton coupled electromagnetic field whose conformal anomaly has been found in [2]. $\phi$ is a complex scalar (the dilaton).

As we intend to include scalars and spinors in our theory, we must add appropriate terms in the action (1). Assuming that there are $M 4 d$ dilaton coupled scalars $X^{k}$, we write

$$
L=f(\operatorname{Re} \phi) g^{\mu \nu} \partial_{\mu} X^{k} \partial_{\nu} X^{k}, \quad k=1, \ldots, M .
$$

Here, $f$ is taken to be some function of the real real part of the dilaton.

Now, as matter Lagrangian we take the one associated with $M$ massless (Dirac) spinors, i.e.

$$
L_{s}=e^{A \phi} \sum_{i=1}^{M} \bar{\psi}_{i} \gamma^{\mu} \nabla_{\mu} \psi^{i} .
$$


It is interesting to note that from the above action one can construct dilatoncoupled Wess-Zumino theory [15]. On a purely bosonic background with only non-zero gravitational and dilaton fields, the conformal anomaly for $\mathcal{N}=4$ super YM theory has been calculated in [6] via AdS/CFT correspondence [5] to be (adding conformal anomaly for dilaton coupled scalar);

$$
\begin{aligned}
T & \left.=b\left(F+\frac{2}{3} \square R\right)+b^{\prime} G+b^{\prime \prime} \square R+C\left[\square \phi^{*} \square \phi-2\left(R^{\mu \nu}-\frac{1}{3} g^{\mu \nu} R\right) \nabla_{\mu} \phi^{*} \nabla_{\nu} \phi\right)\right]+ \\
& +a_{1} \frac{[(\nabla f)(\nabla f)]^{2}}{f^{4}}+a_{2} \square\left(\frac{(\nabla f)(\nabla f)}{f^{2}}\right) .
\end{aligned}
$$

Here

$$
\begin{aligned}
& b=\frac{N^{2}-1}{(4 \pi)^{2}} \frac{N_{s}+6 N_{f}+12 N_{v}}{120}+\frac{M}{120(4 \pi)^{2}}=\frac{N^{2}-1}{4(4 \pi)^{2}}+\frac{M}{120(4 \pi)^{2}}, \\
& b^{\prime}=-\frac{N^{2}-1}{(4 \pi)^{2}} \frac{N_{s}+11 N_{f}+62 N_{v}}{360}-\frac{M}{360(4 \pi)^{2}}=-\frac{N^{2}-1}{4(4 \pi)^{2}}-\frac{M}{360(4 \pi)^{2}}, \\
& C=\frac{N^{2}-1}{(4 \pi)^{2}} N_{v}=\frac{N^{2}-1}{(4 \pi)^{2}} . \\
& a_{1}=\frac{M}{32(4 \pi)^{2}}, \quad a_{2}=\frac{M}{24(4 \pi)^{2}}
\end{aligned}
$$

In the above expression for the anomaly we have taken into account the fact that $N_{s}=6, N_{f}=2, N_{v}=1$ in $\mathcal{N}=4 \mathrm{SU}(\mathrm{N})$ super YM theory; $F=R_{\mu \nu \alpha \beta} R^{\mu \nu \alpha \beta}-2 R_{\mu \nu} R^{\mu \nu}+\frac{1}{3} R^{2}$ is the square of the Weyl tensor in four dimensions; $G$ is the Gauss-Bonnet invariant. The prefactor $N^{2}-1$ appears because all fields are in the adjoint representation. The conformal anomaly for four-dimensional dilaton-coupled scalar has been found in refs. [14.

It is important to note that the anomaly (4) was obtained adding the anomaly for dilaton coupled scalar, but that the terms for the dilaton coupled spinor field were not considered since such an expression would be too complicated to write down explicitly.

Note that both $N$ and $M$ may be considered to be big parameters. So, one can study large- $N$ or large- $M$ expansions.

Let us now find the anomaly induced effective action [7] (for a review, see 
4]). We will write it in non-covariant, local form:

$$
\begin{aligned}
W & =b \int d^{4} x \sqrt{-\bar{g}} \bar{F} \sigma+b^{\prime} \int d^{4} x \sqrt{-\bar{g}}\left[\sigma \left[2 \bar{\square}^{2}+\right.\right. \\
& \left.\left.+4 \bar{R}^{\mu \nu} \bar{\nabla}_{\mu} \bar{\nabla}_{\nu}-\frac{4}{3} \bar{R} \bar{\square}+\frac{2}{3}\left(\bar{\nabla}^{\mu} \bar{R}\right) \bar{\nabla}_{\mu}\right] \sigma+\left(\bar{G}-\frac{2}{3} \bar{\square} \bar{R}\right) \sigma\right]- \\
& -\frac{1}{12}\left[b^{\prime \prime}+\frac{2}{3}\left(b+b^{\prime}\right)\right] \int d^{4} x \sqrt{-\bar{g}}\left[\bar{R}-6 \bar{\square} \sigma-6\left(\bar{\nabla}_{\mu} \sigma\right)\left(\bar{\nabla}^{\mu} \sigma\right)\right]^{2}+ \\
& +C \int d^{4} x \sqrt{-\bar{g}} \sigma \phi^{*}\left(\bar{\square}^{2}+2 \bar{R}^{\mu \nu} \bar{\nabla}_{\mu} \bar{\nabla}_{\nu}-\frac{2}{3} \bar{R} \bar{\square}+\frac{1}{3}\left(\bar{\nabla}^{\mu} \bar{R}\right) \bar{\nabla}_{\mu}\right) \phi+ \\
& +\int d^{4} x \sqrt{-\bar{g}}\left\{a_{1} \frac{[(\nabla f)(\nabla f)]^{2}}{f^{4}} \sigma+a_{2} \square\left(\frac{(\nabla f)(\nabla f)}{f^{2}}\right) \sigma+\right. \\
& \left.+a_{2} \frac{(\nabla f)(\nabla f)}{f^{2}}[(\nabla \sigma)(\nabla \sigma)]\right\} .
\end{aligned}
$$

The computation of the anomaly-induced EA for the dilaton coupled spinor field has been done in [10], and the result, in the non-covariant local form, reads:

$$
\begin{aligned}
W_{s}= & \int d^{4} x \sqrt{-\bar{g}}\left\{\tilde{b} \bar{F} \sigma_{1}+2 \tilde{b^{\prime}} \sigma_{1}\left[\bar{\square}^{2}+2 \bar{R}^{\mu \nu} \bar{\nabla}_{\mu} \bar{\nabla}_{\nu}-\frac{2}{3} \bar{R} \bar{\square}+\frac{1}{3}\left(\bar{\nabla}^{\mu} \bar{R}\right) \bar{\nabla}_{\mu}\right] \sigma_{1}\right. \\
& \left.+\tilde{b}^{\prime} \sigma_{1}\left(\bar{G}-\frac{2}{3} \bar{\square} \bar{R}\right)-\frac{1}{18}\left(\tilde{b}+\tilde{b}^{\prime}\right)\left[\bar{R}-6 \bar{\square} \sigma_{1}-6\left(\bar{\nabla} \sigma_{\mu} \sigma_{1}\right)\left(\bar{\nabla}^{\mu} \sigma_{1}\right)\right]^{2}\right\},
\end{aligned}
$$

Here, $\sigma_{1}=\sigma+A \phi / 3$, for Dirac spinors $\tilde{b}=\frac{3 M}{60(4 \pi)^{2}}, \tilde{b}^{\prime}=-\frac{11 M}{360(4 \pi)^{2}}$.

Moreover, $V_{3}$ is the (infinite) volume of 3 -dimensional flat space, ${ }^{\prime} \equiv d / d \eta$, and $\sigma=\ln a$ where $a(\eta)$ is the scale factor.

Note that in the conformal anomaly (4) we used $g_{\mu \nu}=e^{2 \sigma} \bar{g}_{\mu \nu}$, and all quantities in (5) are calculated with the help of the overbar metric.

Since we know that the anomaly induced effective action is defined with accuracy up to a conformally invariant functional, we may limit ourselves to a conformally flat metric, i. e. $\bar{g}_{\mu \nu}=\eta_{\mu \nu}$. In this case, the conformally invariant functional on a purely gravitational background is zero, and $W+W_{s}$ in Eq. (5) gives the complete contribution to the one-loop effective action. In addition to this we will assume that only the real part of the dilaton coupled to SYM theory is non-zero .

The anomaly induced effective action (5) may now be simplified significantly (due to the fact that $\bar{g}_{\mu \nu}=\eta_{\mu \nu}$ ):

$$
\begin{aligned}
W= & \int d^{4} x\left\{2 b^{\prime} \sigma \square^{2} \sigma-3\left(b^{\prime \prime}+\frac{2}{3}\left(b+b^{\prime}\right)\right) \times\right. \\
& \times\left(\square \sigma+\partial_{\mu} \sigma \partial^{\mu} \sigma\right)^{2}+C \sigma \phi \square^{2} \phi+a_{1} \frac{[(\nabla f)(\nabla f)]^{2}}{f^{4}} \sigma+ \\
& \left.+a_{2} \square\left(\frac{(\nabla f)(\nabla f)}{f^{2}}\right) \sigma+a_{2} \frac{(\nabla f)(\nabla f)}{f^{2}}[(\nabla \sigma)(\nabla \sigma)]\right\},
\end{aligned}
$$


where all derivatives are now flat ones.

Moreover, the anomaly-induced EA for dilaton coupled spinor field, eq. (6), is

$$
W_{s}=V_{3} \int d \eta\left\{2 \tilde{b^{\prime}} \sigma_{1} \sigma_{1}^{\prime \prime \prime \prime}-2\left(\tilde{b}+\tilde{b^{\prime}}\right)\left(\sigma_{1}^{\prime \prime}-\sigma_{1}^{\prime 2}\right)^{2}\right\} .
$$

Considering the case when the scale factor $a(\eta)$ depends only on conformal time: $\sigma(\eta)=\ln a(\eta)$, one has to add the anomaly induced effective action to the classical gravitational action:

$$
S_{c l}=-\frac{1}{\kappa} \int d^{4} x \sqrt{-g}(R+6 \Lambda)=-\frac{1}{\kappa} \int d^{4} x e^{4 \sigma}\left(-6 e^{-2 \sigma}\left(\left(\sigma^{\prime}\right)^{2}+\left(\sigma^{\prime \prime}\right)\right)+6 \Lambda\right),
$$

where $\kappa=16 \pi G$.

We now consider the complete action, $S_{\text {total }}=S_{c l}+W+W_{s}$, and find the gravitational equation of motion by taking the variational derivative with respect to the scale factor $a=e^{\sigma}$. Similarly the field equation of motion is found by taking the variational derivative with respect to $\phi$.

Now, the equations of motion for the action $S_{\text {total }}=S_{c l}+W+W_{s}$, assuming that $\sigma$ and $\phi$ depend only on the conformal time $\eta$, may be written in the following form (assuming the simplest choice $f(\operatorname{Re} \phi)=\phi$ ):

$$
\begin{aligned}
& 24\left(\tilde{b}+\tilde{b}^{\prime}\right)\left(\frac{a^{\prime}}{a}+\frac{A \phi^{\prime}}{3}\right)^{2}-2\left(3 b^{\prime \prime}+2 b+2 \tilde{b}\right) \frac{a^{\prime \prime \prime \prime}}{a}+8\left(3 b^{\prime \prime}+2 b\right) \frac{a^{\prime} a^{\prime \prime \prime}}{a^{2}}+ \\
& +6\left(3 b^{\prime \prime}+2 b+2 \tilde{b}\right) \frac{a^{\prime \prime 2}}{a^{2}}-2\left(3 b^{\prime \prime}+2 b\right)\left(6-\frac{12 b^{\prime}}{3 b^{\prime \prime}+2 b}+\frac{24 \tilde{b}}{3 b^{\prime \prime}+2 b}\right) \frac{a^{\prime \prime} a^{\prime 2}}{a^{3}}- \\
& -24\left(b^{\prime}-\tilde{b}\right) \frac{a^{4}}{a^{4}}+\frac{12}{\kappa} a a^{\prime \prime}-\frac{24 \Lambda}{\kappa} a^{4}-4 \tilde{b} \frac{A \phi^{\prime \prime \prime \prime}}{3}+C \phi \phi^{\prime \prime \prime \prime}+a_{1} \frac{\phi^{\prime}}{\phi^{4}}+a_{2}\left[\frac{\phi^{\prime 2}}{\phi^{2}}\right]^{\prime \prime}- \\
& -2 a_{2}\left(\frac{a^{\prime \prime}}{a}-\frac{a^{\prime 2}}{a^{2}}\right) \frac{\phi^{\prime 2}}{\phi^{2}}-4 a_{2} \frac{a^{\prime}}{a} \frac{\phi^{\prime} \phi^{\prime \prime}}{\phi^{2}}+4 a_{2} \frac{a^{\prime}}{a} \frac{\phi^{\prime 3}}{\phi^{3}}=0, \\
& \frac{A}{3}\left[24\left(\tilde{b}+\tilde{b}^{\prime}\right)\left(\frac{a^{\prime}}{a}+\frac{A \phi^{\prime}}{3}\right)^{2}-4 \tilde{b}\left(\frac{a^{\prime \prime \prime \prime}}{a}-4 \frac{a^{\prime} a^{\prime \prime \prime}}{a^{2}}-3 \frac{a^{\prime \prime 2}}{a^{2}}+12 \frac{a^{\prime 2} a^{\prime \prime}}{a^{3}}-6 \frac{a^{\prime 4}}{a^{4}}+\right.\right. \\
& \left.\left.+\frac{A \phi^{\prime \prime \prime \prime}}{3}\right)\right]+C\left[\ln a \phi^{\prime \prime \prime \prime}+(\ln a \phi)^{\prime \prime \prime \prime}\right]-4 a_{1} \frac{a^{\prime}}{a} \frac{\phi^{\prime 3}}{\phi^{4}}+2 a_{2} \frac{a^{\prime \prime}}{a}\left(\frac{\phi^{\prime 2}}{\phi^{3}}-\frac{\phi^{\prime \prime}}{\phi^{2}}\right)+ \\
& +a_{2}\left(2 \frac{a^{\prime} a^{\prime \prime}}{a^{2}}-\frac{a^{\prime \prime \prime}}{a}\right) \frac{\phi^{\prime}}{\phi^{2}}-12 a_{1}\left[\frac{\phi^{\prime 2} \phi^{\prime \prime}}{\phi^{4}}-\frac{\phi^{\prime 4}}{\phi^{5}}\right] \ln a=0,
\end{aligned}
$$

where $3 b^{\prime \prime}+2 b \neq 0$. The natural choice for $b^{\prime \prime}$ is to take $b^{\prime \prime}=0$, since the choice of $b^{\prime \prime}$ does not make any difference in the physical effects.

Performing the analysis of these equations one may consider some interesting cases. Firstly we may consider case when in our theory we have scalar but 
the spinor is absent. For this problem, the solution was obtained in 12. For this case, an approximate special solution of eqs. (10) may be obtained, when the term with $\ln a$ is negligible. We got $a(t)=a_{0} e^{H t}, \ln a \sim H t$. Moreover, $H$ is proportional to the Planck mass, so $H t$ is small quantity and such an approximation is justified. So using the ansatz (for case of no dilaton it corresponds to anomaly driven inflation [ $[$ ]

$$
a(t) \simeq \tilde{a}_{0} e^{\tilde{H} t}, \quad \phi(t) \simeq \phi_{0} e^{-\alpha \tilde{H} t}
$$

special solutions were found. Since we know that both $N$ and $M$ may be considered to be big parameters, it means one can study large- $N$ or large- $M$ expansions. So in the performed analysis we considered two possibilities for the parameters, namely $N^{2} \approx M$ and $M \gg N^{2}$. It was found that in both cases, the obtained solution was bigger than that obtained in the absence of the dilaton 9] so we conclude that, in this case, the role of the dilaton is to make the inflation faster, as compared with the case of no dilaton.

The second interesting case occurs when we neglect the scalar and take into account only the effect of the spinor. In such a case, as in the case above, one may search for approximate equations of motion (10) (see references [10, 13]), by using the same ansatz (11). From the form of these equations one finds that these solutions will depend on the parameters of the spinor and the dilaton. So one may conclude that such a quantum dS Universe, perturbed by spinors only, may represent the real world if one chooses these parameters appropriately. In fact, related problems were solved in [10, 13]. In these references, by using an ansatz similar to (11), approximate solutions were obtained from which one observes that the value of $H^{2}$ significantly increases by the contribution from the dilaton. One special solution was found explicitly that describes a Brans-Dicke non-singular Universe with a (much slower) expanding dilaton. It is important to note that this is a purely quantum solution which does not exist at the classical level.

Returning to the general theory, i.e. the situation when both scalar and spinor are present, eqs. (10) fully describe our quantum FRW Universe. These equations are too complicated to be solved analytically so it becomes necessary to use some approximation or numerical methods. As in the above cases one may search for special approximate solutions by using the same ansatz (11). As in the two cases before, we find numerical solutions describing a dilatonic dS Universe. Clearly, the parameters of such a Universe are defined by the parameters for the scalar, the spinor, and of course the dilaton. The important lesson of this study is that by a fine tuning of the values of the theory parameters one may find that quantum dS Universe occurs. Such a Universe has attracted interest recently in connection with the dS/CFT correspondence (see [16] for a recent review), related to the current acceleration of the Universe.

Finally, let us make a comment on the form of the coupling function $f$. We assumed above the simplest possible form, $f(\operatorname{Re} \phi)=\phi$. Another simple possibility, assuming $\phi$ to depend only on conformal time, $\phi=\phi(\eta)$, would be 
to assume an exponential form:

$$
f(\eta)=e^{-\beta \eta},
$$

with $\beta$ a constant. With this expression for $f$, the anomaly induced effective action (7) becomes

$$
\begin{aligned}
& W=\int d^{4} x\left\{2 b^{\prime} \sigma \sigma^{\prime \prime \prime \prime}-3\left(b^{\prime \prime}+\frac{2}{3}\left(b+b^{\prime}\right)\right)\left(\sigma^{\prime \prime}+\sigma^{\prime 2}\right)^{2}\right. \\
& \left.+C \sigma \phi \phi^{\prime \prime \prime \prime}+a_{1} \beta^{4} \sigma+a_{2} \beta^{2} \sigma^{\prime 2}\right\} .
\end{aligned}
$$

This form is still analytically tractable. As above, the gravitational equation of motion is found by taking the variational derivative with respect to $a=e^{\sigma}$, whereas the field equation is found by taking the variational derivative with respect to $\phi$.

\section{References}

[1] E. Kolb and M. Turner, The Very Early Universe, Addison-Wesley,NY, 1994

[2] S. Nojiri and S.D. Odintsov, Phys. Lett. B 426, 29 (1998) hep-th 9801052 S. Ichinose and S.D. Odintsov, Nucl. Phys. B 539, 643 (1999) hep-th 9802043

[3] I. Brevik and S.D. Odintsov, Phys. Lett. B455 (1999) 104, hep-th/9902184

[4] I.L. Buchbinder, S.D. Odintsov and I.L. Shapiro, Effective Action in Quantum Gravity, IOP Publishing, Bristol and Philadelphia, 1992.

[5] J.M. Maldacena, Adv. Theor. Math. Phys. 2, 231 (1998), hep-th 9711200

[6] S. Nojiri and S.D. Odintsov, Phys. Lett. B 444, 92 (1998), hep-th 9810008 Phys. Lett. B519, 145 (2001), hep-th/0106191

[7] R. Reigert, Phys. Lett. B 134, 56 (1984); E.S. Fradkin and A. Tseytlin, Phys. Lett. B 134, 187 (1984); I.L. Buchbinder, S.D. Odintsov and I.L. Shapiro, Phys. Lett. B 162, 92 (1985); I. Antoniadis and E. Mottola, Phys. Rev. D 45,2013 (1992); S.D. Odintsov, Z. Phys. C 54, 531 (1992).

[8] A. Starobinsky, Phys. Lett. B 91, 99(1980).

[9] I. Brevik and S.D. Odintsov, "Quantum annihilation of Anti-de Sitter Universe", Phys. Lett. B475 (2000) 247, hep-th/9912032

[10] P. van Nieuwenhuizen, S. Nojiri and S.D. Odintsov, hep-th/9901119 
[11] J. Quiroga and Y. Shaido, Gravitation \& Cosmology ,vol.8,N4 (32) 2002, gr-qc/0206065

[12] J. Quiroga, Mod. Phys. Lett. A, Vol. 18, No. 18 (2003), gr-qc/0305043

[13] B. Geyer, S. D. Odintsov and S. Zerbini, Phys. Lett. B460, 58 (1999), gr-qc/9905073

[14] S. Nojiri and S. D. Odintsov, Phys. Rev. D57 (1998) 2363, hep-th /9706143; Int. J. Mod. Phys. A 16 (2001) 1015, hep-th /0009202.

[15] S. J. Gates, T. Kadoyoshi, S. Nojiri and S. D. Odintsov, Phys. Rev. D58 (1998) 084026, hep-th/9802139

[16] S. D. Odintsov, hep-th/0210094 